\title{
ANÁLISE DOS PRINCIPAIS CONFLITOS E ESPÉCIES INADEQUADAS PRESENTES NA ARBORIZAÇÃO VIÁRIA NA REGIÃO CENTRAL DO MUNICÍPIO DE IMPERATRIZ (MA)
}

\author{
ANALYSIS OF THE MAIN CONFLICTS AND INADEQUATE SPECIES PRESENT IN \\ THE ROAD AFFORESTATION IN CENTRAL REGION OF IMPERATRIZ (MA)
}

Raygo Viana da Silva ${ }^{1}$, Dalton Henrique Angelo², Adriano Araujo Arruda ${ }^{3}$, Wilson Araujo da Silva ${ }^{4}$

\section{RESUMO}

O objetivo do presente trabalho foi avaliar os principais conflitos e espécies inadequadas presentes na arborização urbana da cidade de Imperatriz, a fim de contribuir como ferramenta de gestão para tomada de decisão no planejamento da introdução de espécies vegetais na malha urbana. Com isso, foi realizado um inventário censitário quantitativo da Zona Central e Zona Residencial Central do município de Imperatriz-MA, no qual se coletou informações dos indivíduos arbóreo-arbustivos e da situação do local em que foram implantados. Como resultado, verificou-se que a maioria dos indivíduos se concentra em poucas espécies e foram implantados sem nenhum conhecimento técnico, além de não estarem adequados com a infraestrutura. Constatou-se ainda, que 877 árvores estão em conflitos com a infraestrutura, sendo os mais frequentes os conflitos com imóveis, postes e garagem, geralmente associados às espécies Licania tomentosa Benth. (Fritsch) e Azadirachta indica A. Juss. Recomenda-se o não uso das espécies Licania tomentosa Benth., Azadirachta indica A. Juss., Mangifera indica L., Ficus benjamina L. e Terminalia catappa L. devido a concentração dos indivíduos dessas espécies está acima do satisfatório e por gerar conflitos com a infraestrutura urbana.

Palavras-chave: Levantamento florístico; Planejamento; Elementos urbanos.

\section{ABSTRACT}

The objective of this study was to evaluate the main conflicts and inadequate urban tree species present in the city of Imperatriz, in order to contribute as a management tool for decision making in the planning of the introduction of plant species in the urban network. For that purpose, a quantitative census inventory was carried out in Central and Residential-Central Zones of Imperatriz-MA, collecting data of arboreal and shrub individuals and concerning the location which is inserted. As a result, it has been found that most individuals are distributed into few species and were deployed without any technical knowledge and are not adequate with the infrastructure. It was also verified that 877 trees are in conflicts with the infrastructure, being the most frequent the conflicts with the properties, poles and garage, generally associated to the species Licania tomentosa Benth. (Fritsch) and Azadirachta indica A. Juss. It is recommended not to use the species Licania tomentosa Benth., Azadirachta indica A. Juss., Mangifera indica L., Ficus benjamina L. e Terminalia catappa L., because the concentration of these species is above satisfactory and they generate conflicts with the urban infrastructure.

Key words: Floristic survey; Planning; Urban elements.

\footnotetext{
Recebido em 27.02.2018 e aceito em 03.12.2018

1.Engenheiro Florestal. Graduado pela Universidade Estadual do Maranhão - UEMA. Imperatriz/MA. Email: raygo.florestal@gmail.com

2 Engenheiro Florestal. Me. Professor substituto da Universidade Estadual do Maranhão. Imperatriz/MA. Email: daltonambiente@gmail.com

3 Engenheiro Florestal pela Universidade Estadual da Região Tocantina do Maranhão - UEMASUL. Imperatriz/MA. Email: adriano09arruda@gmail.com

4 Agronomia. Dr. Professor do Departamento de Ciências Agrárias da UEMASUL. Imperatriz/MA. Email: wilsonufrrj@yahoo.com.br
} 


\section{INTRODUÇÃO}

Nos últimos anos tem ocorrido o crescente interesse e necessidade por parte da sociedade, principalmente a comunidade científica, em garantir a promoção da arborização das cidades, visando resgatar a integração do homem com a natureza (PEREIRA, 2015).

A arborização viária contribui de forma considerável para qualidade de vida e bemestar da população, tanto para grandes centros urbanos como pequenas cidades. Dentre os benefícios proporcionados podem ser destacados, a melhoria do microclima, a redução do impacto das chuvas sobre as calçadas e vias, a purificação do ar pela fixação de poeiras, atenuação da poluição sonora, embelezamento das cidades e influência no balanço hídrico (DA FONSECA BORGES; MARIM; RODRIGUES, 2012). Além disso, possibilita a produção de espaços vitais para o abrigo e alimentação dos animais remanescentes no interior das cidades, valoriza o mobiliário urbano, trazendo qualificação ambiental e paisagística dos imóveis, dentre diversos outros (CARVALHO; NUCCI; VALASKI, 2010).

Para Barbosa et al. (2015), pelos benefícios proporcionados, a arborização urbana, deveria ser considerado um dos elementos naturais mais importantes que compõe o ecossistema das cidades, sendo, portanto, primordial no planejamento urbano.

No entanto, em grande parte das cidades brasileiras é notório historicamente as negligências quanto à arborização urbana dentro do planejamento e elaboração de planos diretores das cidades, sendo tratada apenas em sua função ornamental e sem atribuir os benefícios ambientais relevante. Todavia, o poder público tem sido cobrado paulatinamente pelas organizações civis, com objetivo de reconsiderar a conceituação da importância de incluir a arborização dentro do sistema urbano (BRUN et al., 2008).

Por proporcionar diversas vantagens a qualidade de vida da população, é imprescindível o planejamento da arborização de ruas e avenidas, visto que, espécies com porte adequado acarreta diminuição de gastos para sua manutenção, principalmente com podas ao longo do seu desenvolvimento. Salienta-se também que plantas que possuem uma estatura ideal e um distanciamento correto diminuem os conflitos entre suas copas, entre as plantas e a rede elétrica (BARBOSA et al., 2015).

Dessa forma, a arborização urbana deve ser avaliada em seu aspecto quantitativo e qualitativo, sendo indispensável mensuração e o planejamento de modo adequado ao contexto das cidades (COMUNE; SURIANI-AFFONSO, 2014). Para isso, o inventário da arborização viária é o meio mais eficiente e seguro para conhecimento do patrimônio arbóreo-arbustivo da malha urbana, fornecendo informações relacionadas às prioridades de intervenções, possíveis tratamentos fitossanitários, remoção de indivíduos inadequados, bem como o direcionamento para necessidades de manutenção (LIMA NETO; BIONDI, 2014). 
A arborização urbana de ruas e avenidas da cidade de Imperatriz do Maranhão necessita de ferramentas e estratégias que possam subsidiar o planejamento e o manejo das espécies arbóreas presentes na malha viária, visto que, não tão obstante da maioria das cidades brasileiras, se apresenta de forma irregular, inadequada e consequentemente conflitante com os elementos da infraestrutura urbana.

Por conta disso, objetivou-se com esse trabalho avaliar os principais conflitos e espécies inadequadas presentes na arborização urbana da cidade de Imperatriz, a fim de auxiliar como ferramenta de gestão para tomada de decisão no planejamento da introdução de espécies vegetais na malha urbana.

\section{MATERIAL E MÉTODOS}

\section{Área de Estudo}

O estudo ocorreu na cidade Imperatriz, localizada no Sudoeste do Maranhão

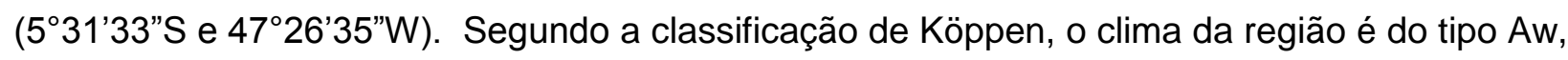
ou seja, tropical quente e úmido com duas estações bem definidas, que corresponde um período de chuva que vai de dezembro a abril, e um período seco de maio a novembro, apresentando uma temperatura média anual em torno de $29{ }^{\circ} \mathrm{C}$ (PREFEITURA DE IMPERATRIZ, 2016). Na figura 1 é apresentada a localização da área de estudo.

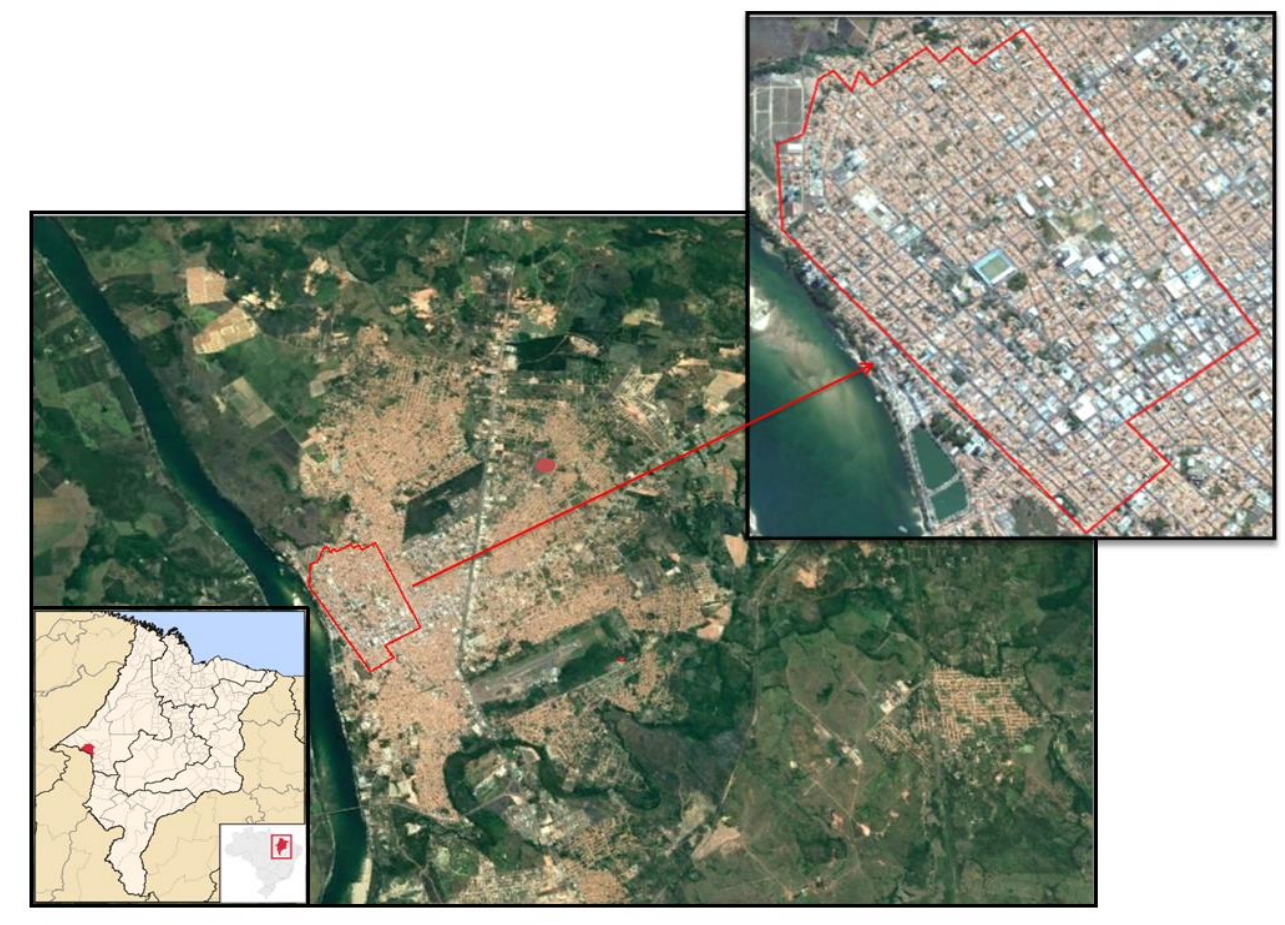

Fonte: Google Earth (2016)

Figura 1. Vista geral e localização da área de estudo no município de Imperatriz, MA

Figure 1. Overview and location of the analyzed area in the city of Imperatriz, MA 
A cidade conta atualmente com uma população estimada de 253.873 habitantes, ou seja, apresenta uma densidade demográfica de 180,79 hab . km-1, que a classifica como a segunda maior cidade do Estado do Maranhão (IBGE, 2016).

\section{Procedimentos Metodológicos}

O inventário quali-quantitativo censitário foi realizado na Zona Central (ZC) e Zona Residencial Central (ZRC), definidas de acordo com a Lei № 003/2004, que dispõe do zoneamento, parcelamento, uso e ocupação dos solos do município de Imperatriz (figura 2). A divisão em Zonas objetiva a avaliação de forma pontual das diferentes situações encontradas dentro da região de estudo.

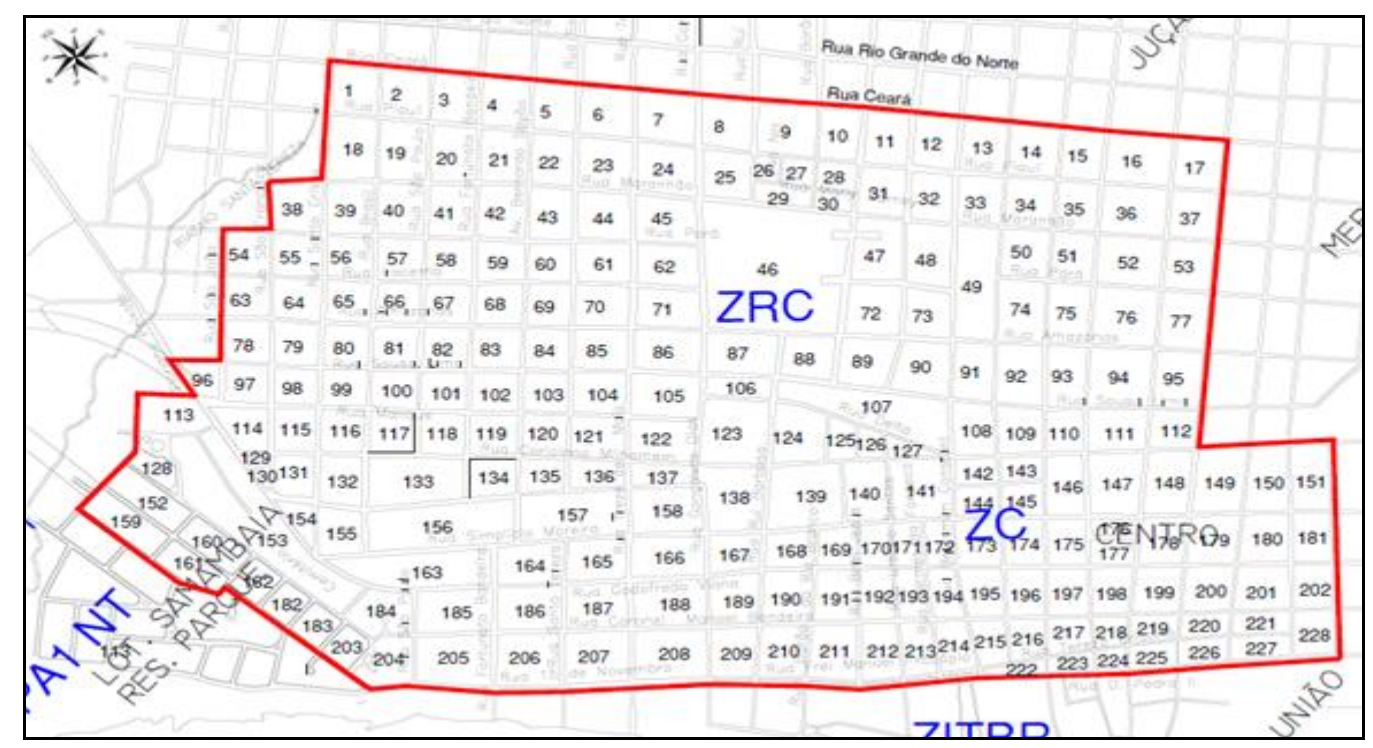

Fonte: Prefeitura de Imperatriz, 2014.

Figura 2. Delimitação da área de estudo segundo a Lei de Zoneamento 003/2004

Figure 2. Delimitation of the study área according to the Zoning Law 003/2004

O período do levantamento das informações em campo foi de fevereiro de 2016 a fevereiro de 2017, em que foram coletados dados referentes ao patrimônio arbóreo-arbustivo presentes nas vias públicas das 228 quadras localizadas nos limites das ZC e ZRC, além da infraestrutura no qual as árvores estão inseridas.

Foram catalogadas todas as espécies presentes nas ruas e avenidas e coletadas informações dendrométricas de árvores e arbustos com altura superior a 1,5 m e apenas contabilizado os arbustos e mudas com altura inferior ao parâmetro definido. No levantamento foi coletada a Circunferência a Altura do Peito (CAP a 1,30 m de altura) que posteriormente foi convertida em Diâmetro a Altura do Peito (DAP a 1,30 $\mathrm{m}$ de altura) através da fórmula: DAP = $\mathrm{CAP} / 3,14$. 
Com isso, os dados coletados em campo foram referentes ao: tipo de espécie (nome vulgar e científico), a altura total, o diâmetro à altura do peito, a altura de bifurcação, a altura e a área da copa, a área disponível ao seu desenvolvimento, se há a presença de injúrias (pragas, doenças ou danos provocados pela ação do homem), qualidade de poda e aspecto paisagístico.

Quanto aos parâmetros físicos (estrutura física), realizou-se um levantamento das características relacionadas à interação das árvores com os elementos urbanos, para isso, foram coletadas informações referentes ao espaçamento entre espécies; largura e comprimento das calçadas; distância da árvore para a construção e meio fio; se há ou não algum conflito e o tipo (poste, placa, construção, vias, garagem, marquises, fiação, esquina ou rede de esgoto); altura da fiação; e as condições físicas com pavimento após a inserção da espécie (se há danos provocados pelas raízes).

Para identificação das espécies foram utilizadas bibliografias especializadas com relação das famílias botânicas e suas respectivas espécies. Quando não foi possível encontrar as espécies nessas bibliografias, utilizou-se da lista de espécies da flora brasileira Programa de Reconhecimento da Flora Brasileira (REFLORA), levando sempre em consideração o sistema APGIII.

Todos os dados após serem coletados em campo foram reunidos em uma planilha do Software Excel, posteriormente, prosseguiu-se com as análises.

Os principais conflitos avaliados foram: conflito com poste (POS); conflito com a sinalização (SIN); conflitos com garagem (GAR), conflitos com a esquina (ESQ); e conflito com a Fossa (FOS). Após o levantamento, calculou-se a frequência relativa dos conflitos dentro da malha urbana, através do número de indivíduos conflitantes da espécie sobre o número total de árvores multiplicada por 100.

Além disso, com relação à interação das árvores e arbustos com a rede de distribuição de energia, ordenou-se os indivíduos em 3 classes: a classe $A$ encontra-se todos os indivíduos que não apresentaram conflitos com a rede de distribuição de energia, porém não é correto dizer que futuramente estes não poderão causar algum tipo de interferência a rede elétrica; a classe $B$ reúne os indivíduos nos quais a rede elétrica atravessa a copa; e a classe C apresenta os indivíduos que a rede elétrica está acima de sua copa.

As espécies foram reunidas também em classes de diâmetro e altura para melhor tratamento dos dados e análise do porte. Com isso, foram classificados os indivíduos em quatro classes de diâmetro, sendo: A (0,15 a 14, $99 \mathrm{~cm})$; B (15 a 29,99 cm); C (30 a 44,99 cm); e D $(45$ a 59,99); e E (acima de $60 \mathrm{~cm})$. Já as alturas dos indivíduos foram reunidas em 3 classes, sendo: $F(1,5>4,4 \mathrm{~m})$; $\mathrm{G}(4,5 \geq 5,4 \mathrm{~m})$; e H $(\geq 5,5 \mathrm{~m})$. 


\section{RESULTADO E DISCUSSÃO}

Dentre as 228 quadras inventariadas e 891,4487 km percorridos de calçada, foram encontrados 2321 indivíduos arbóreo-arbustivos presentes em ruas e avenidas da cidade de Imperatriz, sendo catalogados na Zona Central (ZC) e Zona Residencial Central (ZRC), respectivamente, 752 e 1569 indivíduos.

No levantamento foram catalogadas 69 espécies, enquadradas em 27 famílias, sendo as cinco mais representativas: Fabaceae (17 espécies), Apocynaceae (6 espécies), Myrtaceae (6 espécies), Rutaceae (5 espécies) e Bignoneaceae (4 espécies). Na tabela 1 estão listadas todas as espécies encontradas na área de estudo e as respectivas frequências.

Tabela 1. Frequência de espécies arbóreo-arbustivas encontradas nas vias públicas de Imperatriz, MA Table 1. Frequency of arboreal-shrub species found on public roads in Imperatriz, MA

\begin{tabular}{|c|c|c|c|c|c|}
\hline Família & Nome Popular & Nome Científico & 0 & № & $\begin{array}{l}\text { FR } \\
(\%)\end{array}$ \\
\hline \multirow{3}{*}{ Anacardiaceae } & Cajazeiro & Spondias mombin $L$. & $\mathrm{N}$ & 3 & 0,13 \\
\hline & Cajueiro & Anacardium o & $\mathrm{N}$ & & 0,39 \\
\hline & Mangueira & Mangifera & $\mathrm{E}$ & 89 & 3,83 \\
\hline \multirow{2}{*}{ Annonaceae } & Ata & Annona squamosa L. & $\mathrm{E}$ & 14 & 0,60 \\
\hline & Pimenta-de-Macaco & Xylopia aromatica (Lam.) Mart. & $\mathrm{N}$ & 1 & 0,04 \\
\hline \multirow{6}{*}{ Apocynaceae } & Alamanda & Allamanda cathartica L. & $\mathrm{N}$ & 1 & 0,04 \\
\hline & Chapéu-de- & Thevetia peruviana (Pers.) K. Schum & $\mathrm{E}$ & 5 & 0,22 \\
\hline & Espirradeira & Nerium oleander $\mathrm{L}$. & $\mathrm{E}$ & 4 & 0,17 \\
\hline & & pis procera (Aiton) W. & $\mathrm{E}$ & 1 & 0,04 \\
\hline & & Plumeria rubra L. & $\mathrm{E}$ & 11 & 0,47 \\
\hline & Jasn & Plume & $\mathrm{E}$ & 18 & 0,78 \\
\hline \multirow{4}{*}{ Bignoniaceae } & Ipê-Amarelo & Handroanthus serratifolius (Vahl) S.O.Grose & $\mathrm{N}$ & 49 & 2,11 \\
\hline & & Tecoma stans (L.) Juss. ex Kunth & $\mathrm{E}$ & 17 & 0,73 \\
\hline & & indroanthus & $\mathrm{N}$ & 5 & 0,22 \\
\hline & Spathode & Spathodea & $\mathrm{E}$ & 1 & 0,04 \\
\hline Caesalpiniaceae & Caneleiro & Cenostigna macrophyllum Tul. & $\mathrm{N}$ & 5 & 0,22 \\
\hline Caric & Mamão & Carica papaya L. & $\mathrm{E}$ & 2 & 0,09 \\
\hline Caryc & Pequi & Caryocark & $\mathrm{N}$ & 2 & 0,09 \\
\hline Chrys & & Licania ton & $\mathrm{N}$ & 1150 & 49,55 \\
\hline Clus & Clusia-Sans & Clusia burchellii Engl. & $\mathrm{N}$ & 1 & 0,04 \\
\hline Combretaceae & Amendoeira & Terminalia catappa $L$. & $E$ & 67 & 2,89 \\
\hline Combre & Jasmim-da-Índia & Quisqualis indica L. & $E$ & 27 & 1,16 \\
\hline Dilleniaceae & Árvore-do-Dinheiro & Dillenia indica $L$. & $\mathrm{E}$ & 6 & 0,26 \\
\hline Euphorbiaceae & Pião-Roxo & Jatropha gossypiifolia L. & $\mathrm{E}$ & 6 & 0,26 \\
\hline \multirow{7}{*}{ Fabaceae } & $\begin{array}{l}\text { Pinhão-Branco } \\
\text { Angico-Vermelho }\end{array}$ & \multirow{7}{*}{$\begin{array}{c}\text { Anadenanthera macrocarpa (Benth.) Brenan } \\
\text { Chloroleucon tenuiflorum (Benth.) Barneby } \\
\text { \& J. W. Grimes } \\
\text { Erythrina variegata L. } \\
\text { Senna siamea (Lam.) H. S. Irwin \& } \\
\text { Barneby } \\
\text { Cassia fistula L. } \\
\text { Adenanthera pavonina L. } \\
\text { Senna pendula var. ambigua H.S. Irwin \& } \\
\text { Barneby } \\
\text { Delonix regia (Bojer ex Hook.) Raf. }\end{array}$} & $\frac{E}{N}$ & 2 & $\begin{array}{l}0,09 \\
0,09\end{array}$ \\
\hline & Barreiro & & $\mathrm{N}$ & 3 & 0,13 \\
\hline & Brasileirinho & & $E$ & 6 & 0,26 \\
\hline & Cássia-de-Sião & & $E$ & 5 & 0,22 \\
\hline & $\begin{array}{c}\text { Chuva-de-Ouro } \\
\text { Carolina }\end{array}$ & & $\begin{array}{l}E \\
E\end{array}$ & $\begin{array}{l}6 \\
7\end{array}$ & $\begin{array}{l}0,26 \\
0,30\end{array}$ \\
\hline & Fedegoso & & $\mathrm{N}$ & 14 & 0,60 \\
\hline & Flamboyant & & $\mathrm{E}$ & 2 & 0,09 \\
\hline
\end{tabular}




\begin{tabular}{|c|c|c|c|c|c|}
\hline \multirow[t]{9}{*}{ Família } & Nome Popular & Nome Científico & 0 & № & $\begin{array}{l}\text { FR } \\
(\%)\end{array}$ \\
\hline & Ingá-Doce & Pithecellobium dulce (Roxb) Benth & $E$ & 19 & 0,82 \\
\hline & Ingá-Cipó & Inga edulis Mart & $\mathrm{N}$ & 1 & 0,04 \\
\hline & Pata-de-Vaca & Bauhinia forficata Benth & $\mathrm{N}$ & 6 & 0,26 \\
\hline & Pau-Brasil & Caesalpinia echinata Lam. & $\mathrm{N}$ & 3 & 0,13 \\
\hline & Pau-Ferro & Caesalpinia ferrea Mart. ex Tul. & $\mathrm{N}$ & 2 & 0,09 \\
\hline & Sibipiruna & Caesalpinia pluviosa DC. & $\mathrm{N}$ & 21 & 0,90 \\
\hline & Sombreiro & $\begin{array}{c}\text { Clitoria fairchildiana R.A. Howard } \\
\text { R.A.Howard }\end{array}$ & $\mathrm{N}$ & 13 & 0,56 \\
\hline & Tamarindo & Tamarindus indica L. & $E$ & 6 & 0,26 \\
\hline Lamiaceae & Teca & Tectona grandis L. f. & $\mathrm{E}$ & 1 & 0,04 \\
\hline Lythraceae & Extremosa & Lagerstroemia indica L. & $\mathrm{E}$ & 16 & 0,69 \\
\hline Malpighiaceae & Acerola & Malpighia glabra L. & $E$ & 7 & 0,30 \\
\hline \multirow{2}{*}{ Malvaceae } & Hibisco & Hibiscos sinensis Mill. & $\mathrm{E}$ & 1 & 0,04 \\
\hline & Monguba & Pachira aquatica Aubl. & $\mathrm{N}$ & 55 & 2,37 \\
\hline Meliaceae & Nim-indiano & Azadirachta indica A. Juss. & $\mathrm{E}$ & 333 & 14,34 \\
\hline \multirow{3}{*}{ Moraceae } & Amora & Morus nigra L. & $E$ & 3 & 0,13 \\
\hline & Ficus & Ficus benjamina (L.) & $E$ & 137 & 5,90 \\
\hline & Jaca & Artocarpus heterophyllus Lam. & $\mathrm{E}$ & 2 & 0,09 \\
\hline \multirow{6}{*}{ Myrtaceae } & Eucalipto & Eucalyptus sp. & $E$ & 1 & 0,04 \\
\hline & Goiabeira & Psidium guajava L. & $\mathrm{E}$ & 10 & 0,43 \\
\hline & Jabuticabeira & Plinia trunciflora (O. Berg) Kausel & $\mathrm{N}$ & 1 & 0,04 \\
\hline & Jambo-Vermelho & Eugenia malaccensis (L.) & $E$ & 25 & 1,08 \\
\hline & Jambo-Branco & Syzygium aqueum (Burm. f.) Alston & $E$ & 1 & 0,04 \\
\hline & Jamelão & Syzygium cumini (L.) Skeels & $\mathrm{E}$ & 20 & 0,86 \\
\hline Nictaginaceae & Primavera & $\begin{array}{l}\text { Bougainvillea spectabilis var. glabra } \\
\text { (Choisy) Hook. }\end{array}$ & $\mathrm{N}$ & 3 & 0,13 \\
\hline Rhamnaceae & Juá & Ziziphus joazeiro Mart. & $\mathrm{N}$ & 1 & 0,04 \\
\hline Rubiaceae & Noni & Morinda citrifolia L. & $E$ & 2 & 0,09 \\
\hline \multirow{5}{*}{ Rutaceae } & Laranjeira & Citrus sinensis (L.) Osbeck & $E$ & 10 & 0,43 \\
\hline & Lima & Citrus limettioides Tanaka & $E$ & 2 & 0,09 \\
\hline & Limão & Citrus limon (L.) Osbeck & $E$ & 7 & 0,30 \\
\hline & Murta & Murraya paniculata (L.) Jack & $E$ & 54 & 2,33 \\
\hline & Tangerina & Citrus reticulata Blanco & $\mathrm{E}$ & 1 & 0,04 \\
\hline \multirow[t]{2}{*}{ Salicaceae } & Salgueiro-Chorão & Salix babylonica L. & $\mathrm{E}$ & 2 & 0,09 \\
\hline & Maria-Mole & Dilodendrom bipinnatum Radlk & $E$ & 2 & 0,09 \\
\hline \multirow[t]{2}{*}{ Sapindaceae } & Pitomba & Talisia esculenta (A. St.-Hil.) Radlk. & $\mathrm{N}$ & 1 & 0,04 \\
\hline & Sapoti & Manilkara zapota (L.) P. Royen & $\mathrm{E}$ & 6 & 0,26 \\
\hline 27 & & 69 & & 21 & $100 \%$ \\
\hline
\end{tabular}

Observou-se que as espécies que apresentaram maior número de indivíduos foram Licania tomentosa Benth. (Fritsch) com 49,55\% (1150 indivíduos), Azadirachta indica A. Juss. 14,34 \% (333 indivíduos), Ficus benjamina L. 5,90\% (137 indivíduos), Mangifera indica L. 3,83\% (89 indivíduos), Murraya paniculata (L.) Jack. 2,33\% (54 indivíduos) e Terminalia catappa L. 2,57\% (67 indivíduos) (Tabela 1).

Constatou-se ainda, que a arborização é representada por muitos indivíduos concentrados em poucas espécies, sendo prejudicial à qualificação da arborização da cidade, uma vez que uma grande concentração de indivíduos de uma mesma espécie pode contribuir para a propagação de pragas e doenças nas árvores, devida à facilidade de disseminação dos agentes transmissores. 
Morais e Machado (2014) evidenciam que a implantação de uma mesma espécie em grandes proporções se relaciona a facilidade de aquisição das mudas, a boa adaptação da árvore no ambiente das cidades e o desconhecimento da população à respeito da biologia da espécie. Tal fato pode ser verificado na cidade de Imperatriz com a introdução de forma exorbitante das espécies Licania tomentosa Benth. (Fritsch) (49,55\%) e Azadirachta indica A. Juss. (14,34\%), sendo evidenciadas de maneira predominante tanto na ZC como na ZRC, atribuída a facilidade de aquisição das mudas (tabela 1).

A situação da região central de Imperatriz não se distancia das demais cidades brasileiras, Sampaio e De Angelis (2008), verificaram que em Maringá-PR a espécie mais representativa foi Caesalpinia peltophoroides Benth. com 39,21\% do total avaliados de 69727 indivíduos. Já em Jataí no estado do Goiás verificou que Licania tomentosa Benth. (Fritsch) predomina na arborização, perfazendo $38,3 \%$ do total de indivíduos (BARROS; GUILHERME; CARVALHO, 2010).

Verificou-se que de um total de 69 espécies encontradas, 46,38\% (32 espécies) podem ser consideradas de grande porte (podem atingir altura superior a 10 metros), 33,33\% (23 espécies) de pequeno porte (altura entre 4 a 6 m) e 20,29\% (14 espécies) de médio porte (alturas entre 6 a 10 metros), de acordo com a classificação de Mascaró e Mascaró, (2005). O porte das espécies é uma variável importante, uma vez que indivíduos com altura superior a 5 $\mathrm{m}$ alcança a rede de serviços aérea (rede elétrica ou de telefonia) e está sujeita às podas de condução pelas concessionárias, sendo este um processo que deforma os vegetais e, em muitas situações, compromete sua integridade e desenvolvimento (SALVI et al., 2011).

No tocante ao desenvolvimento vegetal dos indivíduos, constatou-se que a maioria dos indivíduos arbóreo-arbustivos se concentram nas classes de menores diâmetros, tanto na ZC como na ZRC, sendo representado respectivamente por $81,17 \%$ e $92,36 \%$ nas Classes $\mathrm{A}$ e $\mathrm{B}$ em relação ao total de indivíduos para cada zona (tabela 2).

Tabela 2. Distribuição de frequência dos indivíduos arbóreo-arbustivos em diferentes classes de diâmetros na Zona Central (ZC) e Zona Residencial Central (ZRC)

Table 2. Frequency distribution of arboreal-shrub individuals in different diameter classes in Central Zone (ZC) and Central Residential Zone (ZRC)

\begin{tabular}{ccccc}
\hline \multirow{2}{*}{ Intervalo de DAP (cm) } & \multicolumn{2}{c}{ ZC } & \multicolumn{2}{c}{ ZRC } \\
\cline { 2 - 5 } & № Indivíduos & FR(\%) & № Indivíduos & FR(\%) \\
\hline A (1,5 - 14,99) & 366 & 48,54 & 954 & 60,84 \\
B (15 - 29,99) & 246 & 32,63 & 495 & 31,57 \\
C (30 - 44,99) & 79 & 10,48 & 82 & 5,23 \\
D (45 - 59,99) & 36 & 4,77 & 30 & 1,91 \\
E ( $\geq \mathbf{6 0})$ & 27 & 3,58 & 7 & 0,45 \\
Total & 754 & 100 & 1568 & 100 \\
\hline
\end{tabular}

Nota: ZC - Zona Central; ZRC - Zona Residencial Central; FR(\%) - Frequência Relativa. 
A porcentagem de exemplares presentes nas Classes $C$, D e E perfaz um total de $26,47 \%$, sendo observados que a maioria dos indivíduos que apresentavam diâmetro maior, apontavam algum tipo de conflito com as infraestrutura urbana, pois em sua maioria eram classificadas como de médio ou grande porte.

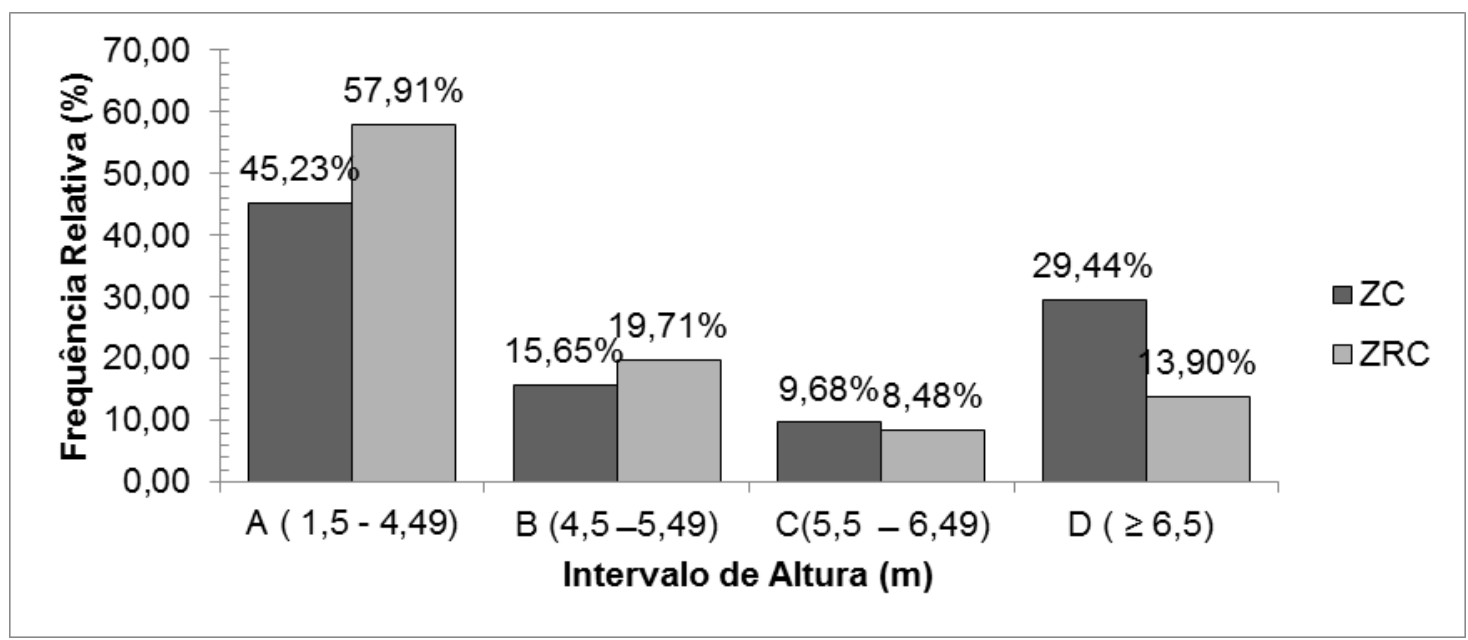

Figura 2. Distribuição dos indivíduos em diferentes classes de altura

Figure 2. Distribution of individuals in different height classes

O número de indivíduos concentrados na Classe $A$ de altura é maior que as demais classes, tanto para ZC como ZRC, representando 45,23\% (366 indivíduos) e 57,91\% (954 indivíduos), respectivamente. Em comparação com altura da rede elétrica média encontrada na cidade de Imperatriz (4,9 m com desvio padrão $\pm 1,09 \mathrm{~m}$ ), constata-se que as classes $B, C$ e D, podem apresentar algum tipo de conflito com a rede elétrica, devido a espécie não ter uma altura compatível com locais que possuem rede de distribuição de energia (figura 2).

Cabe ressaltar ainda, que fatores como clima, ambiente urbano e podas frequentes, podem contribui para o baixo desenvolvimento das espécies em altura, pois uma vez fora do ambiente natural o comportamento fisiológico da árvore pode ser alterado. Melo, Lira Filho e Junior (2007) verificaram que mais de $90 \%$ dos indivíduos apresentavam altura inferior a $5 \mathrm{~m}$ atribuída ao desenvolvimento das espécies ou as interferências do clima no processo de crescimento.

Vale lembrar que espécies que foram introduzidas em calçadas sem nenhum planejamento prévio quanto a sua adequação com a infraestrutura do local podem acarretar diferentes tipos de conflitos, tendo como consequência a adoção de podas, principalmente para impedir contato com a fiação aérea, apresentando assim, árvores com altura reduzida (DE SOUZA et al., 2014).

Na região central de Imperatriz, verificou-se que $81,65 \%$ dos indivíduos inventariados apresentava algum tipo de poda, sendo mais frequente principalmente na ZRC $(53,94 \%)$, 
devido à maior quantidade de indivíduos presentes. Do total analisado, 60,79\% (1411 indivíduos) apresentaram podas leves, 15,21\% (381 indivíduos) poda pesada e 5,64\% (131 indivíduos) poda drástica.

Constatou-se também, que 1164 indivíduos foram plantados em calçadas que dão suporte a rede elétrica, em 28,9\% destes indivíduos (336 árvores), a rede atravessa a copa, sendo mais expressivo na ZRC do que na ZC, sendo respectivamente 19,1\% (222 árvores) e 9,8\% (114 árvores).

Além disso, diversos indivíduos poderão futuramente exercer algum tipo de conflito com a fiação, pois apesar de 62,6\% (729 indivíduos) das árvores apresentarem a rede aérea acima de suas copas, espécies como Licania tomentosa Benth. (Fritsch.) e Azadirachta indica Benth. (Fritsch.) consideradas de grande porte, representam 27,2\% do total de árvores com copa abaixo da fiação, o que poderá ser uma ameaça futura a rede de distribuição de energia caso não seja aplicado algum tipo de manejo dessas espécies.

Almeida e Barbosa (2010), verificaram no diagnóstico do patrimônio arbóreo de ruas e avenidas de Cocoal-RO, que $56,10 \%$ das árvores avaliadas apresentavam copas que tocavam a fiação elétrica, pondo em risco a vida humana, principalmente em dia chuvoso por provocar descargas elétricas.

Dentre todo o patrimônio arbóreo-arbustivo avaliado, observou-se que 1468 indivíduos, não necessitam de nenhum tipo de poda, pois já haviam sido podadas, representando mais de $63 \%$ do total inventariado. Todavia, 1009 árvores precisam ser podadas para mitigar os conflitos com a construção, rede de distribuição de energia, levantamento de copa e condução, principalmente na ZRC (Tabela 2).

Avaliando a necessidade de poda por Zonas, verifica-se uma maior necessidade de condução, afastamento de construção e equilíbrio para as árvores da ZRC, perfazendo, 21,54\% (500 árvores), 14,00\% (325 árvores) e 8,10\% (188 árvores), respectivamente. Já a ZC, o levantamento de copa se faz mais necessário, representando 6,89\% (160 árvores) do total. Tal fato pode ser explicado pela falta de planejamento na escolha e tratos culturais com as espécies que são implantadas em ruas e avenidas da cidade (Tabela 3).

Do total de árvores avaliadas na ZC e ZRC, 877 indivíduos apresentavam algum tipo de conflito com a infraestrutura urbana, representando $37,78 \%$ do total de árvores presentes na arborização urbana da região central de Imperatriz. Na tabela 4, nota-se que 59,41\% (521 árvores) envolve interferências sobre a construção devido a falta de condução e manutenção das espécies implantadas em calçadas. Além disso, verifica-se que 15,05\% (132 árvores) conflitam com poste, 12,20\% (107 árvores) obstrui o acesso à garagem e 4,22\% (37 árvores) retira a visibilidade da sinalização, conflitos com a marquise e sinalização se igualam com 3,31\% (29 árvores) cada um. 
Tabela 3. Indivíduos com necessidade de poda encontrados em vias públicas da cidade de Imperatriz, MA

Table 3. Individuals in need of pruning found on public roads in the city of Imperatriz, MA

\begin{tabular}{|c|c|c|c|c|}
\hline \multicolumn{5}{|c|}{ Necessidade de Poda } \\
\hline \multirow{2}{*}{ Tipo de Poda } & \multicolumn{2}{|c|}{ ZC } & \multicolumn{2}{|c|}{ ZRC } \\
\hline & № & FR(\%) & № & FR(\%) \\
\hline Ausente & 308 & 13,27 & 545 & 23,48 \\
\hline Afastamento da construção & 204 & 8,79 & 325 & 14,00 \\
\hline Liberação de rede & 69 & 2,97 & 103 & 4,44 \\
\hline Levantamento de copa & 160 & 6,89 & 106 & 4,57 \\
\hline \multirow{2}{*}{ Condução } & 71 & 3,06 & 500 & 21,54 \\
\hline & 11 & 0,47 & 2 & 0,09 \\
\hline Limpeza & 13 & 0,56 & 3 & 0,13 \\
\hline Equilíbrio & 72 & 3,10 & 188 & 8,10 \\
\hline Afastamento de placa & 10 & 0,43 & 4 & 0,17 \\
\hline Afastamento semáforo & 1 & 0,04 & 0 & 0,00 \\
\hline Outros & 32 & 1,38 & 13 & 0,56 \\
\hline \multicolumn{5}{|c|}{$\begin{array}{l}\text { Tabela 4. Ocorrência dos principais conflitos encontrados na arborização urbana da cidade d } \\
\text { Imperatriz, MA }\end{array}$} \\
\hline Table 4. Occurrence of the main conflict & ounds in the urban a & ation & ity of & riz \\
\hline Tipo & № de ocorrências & \multicolumn{3}{|c|}{ FR (\%) } \\
\hline Construção & 521 & \multicolumn{3}{|c|}{59,41} \\
\hline \multirow{2}{*}{$\begin{array}{l}\text { Poste } \\
\text { Sinalizacão }\end{array}$} & 132 & \multicolumn{3}{|c|}{15,05} \\
\hline & 37 & \multicolumn{3}{|c|}{4,22} \\
\hline Garagem & 107 & \multicolumn{3}{|c|}{12,20} \\
\hline Esquina & 29 & \multicolumn{3}{|c|}{3,31} \\
\hline Marquise & 29 & \multicolumn{3}{|c|}{3,31} \\
\hline Fossa & 1 & & 0 & \\
\hline Outros & 21 & & 2, & \\
\hline Total & 877 & & 100 & \\
\hline
\end{tabular}

Quando avaliados os três conflitos mais frequentes em relação às espécies mais representativas dentro da arborização, observa-se que Licania tomentosa Benth. (Fritsch.) e Azadirachta indica A. Juss. apresentam 45,15\% e 12,43\%, respectivamente, do total de conflitos presentes na arborização de Imperatriz, estes valores estão associados ao plantio sem adotar critérios técnicos, realizado pelos próprios moradores (figura 4).

A compressão da interação do espaço urbano com as áreas verdes e suas consequências tornam-se vital quando se pretendem adequar projetos que valorizem o ambiente natural, sendo fundamental para atribuir mais qualidade de vida aos citadinos (DA FONSECA BORGES; MARIM; RODRIGUES, 2012). Com isso, é essencial a adoção correta e criteriosa de um planejamento prévio, em que sejam levados em consideração fatores que influenciam na escolha das espécies, na produção das mudas e implantação das mesmas (BOBROWSKI, 2011). Desse modo, todas as estratégias empregadas no planejamento da arborização, devem ser fundamentadas em informações técnicas que se baseiam nas possibilidades do cenário físico do local escolhido para implantação das espécies. 
Outra parte fundamental no gerenciamento das áreas verdes na malha urbana é a análise qualitativa das árvores, que desempenha a função de prever o surgimento de conflitos, identificar os atuais e propor soluções adequadas às características do local. Sendo assim, é indispensável à elaboração de planos para a manutenção dessas áreas, pois não seria útil ter projetos bem elaborados e bem executados se este não for mantido ao longo do tempo (SANTOS et al., 2015).

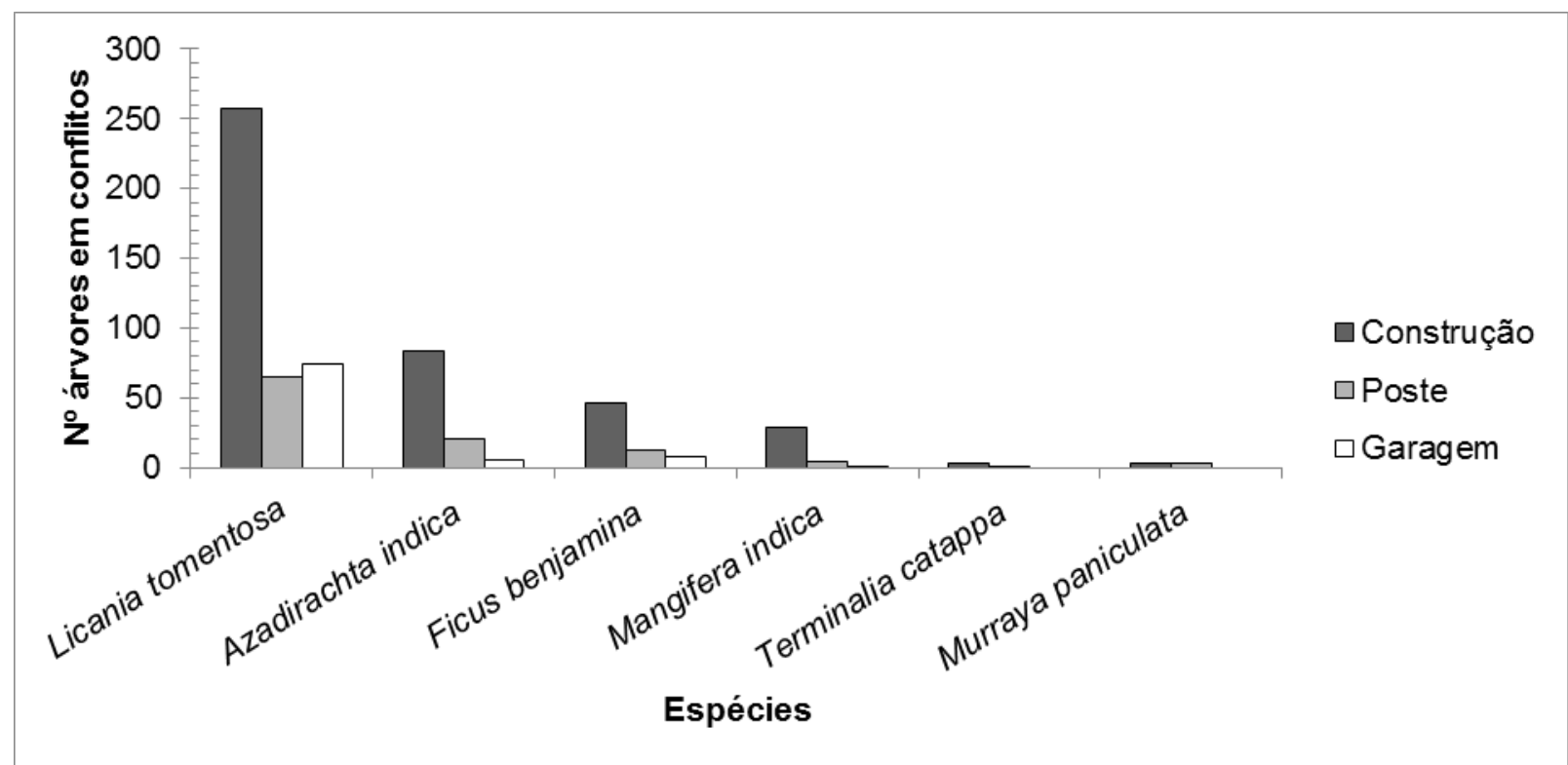

Figura 3. Relação entre os três principais conflitos e as espécies envolvidas na arborização urbana de Imperatriz, MA

Figure 3. Relation between the three main conflicts and the species involved in the urban afforestation of Imperatriz, MA

No que diz respeito à área livre disponível ao desenvolvimento das espécies encontradas nas calçadas da cidade de Imperatriz, obteve-se uma média de 0,26 $\mathrm{m}^{2}$ com desvio padrão de $\pm 0,43 \mathrm{~m}^{2}$, denotando uma área livre insuficiente, sendo recomendado uma área superior a $1 \mathrm{~m}^{2}$ (BORTOLETO; FILHO; LIMA, 2006). Das 2321 árvores e arbustos avaliados, apenas 1,08\% (25 árvores) possuem área livre acima de $1 \mathrm{~m}^{2}$, o que impede 0 desenvolvimento das espécies, além de causar conflitos e danos ao pavimento.

Esse problema é bastante comum nas cidades brasileiras, como foi apontado por Paiva (2009) no município de Cosmopolis-SP, em que a área livre apresentou média de 0,5 $\mathrm{m}^{2}$, o que agrava a situação caso a espécies seja inadequada, visto que a ação mecânica do crescimento secundário do tronco das árvores extrapola área livre sem pavimento.

Ao avaliar as condições das raízes no pavimento, notou-se que $85,35 \%$ das árvores não causam interferência ao passeio, 5,55\% a raiz se apresenta superficial, mas não danificam a calçada, 7,45\% quebram a calçada e 1,64\% destrói o pavimento. Apesar da maioria dos 
indivíduos estarem ausentes de conflitos com o pavimento, não se descarta a hipótese de que futuramente sejam ocasionados danos ao calçamento presente na cidade.

Ao se conhecer as espécies, bem como o seu sistema radicular, torna-se uma análise fundamental para se evitar transtornos proporcionados pelo afloramento de raízes superficiais, pois um sistema radicular pouco profundo ocasiona destruição das calçadas e canteiros, que, muitas vezes, comprometem a estrutura dos imóveis (SANTOS et al., 2015).

Quando se avalia as espécies que contribuíram para o afloramento de raiz que proporcionou danos ao pavimento, os exemplares que mais se envolveram em conflitos com o a calçada foram Licania tomentosa Benth. (Fritsch) (105 indivíduos), Maginfera indica L. (53 indivíduos), Azadirachta indica (45 indivíduos), Terminalia catappa (36 indivíduos) e Ficus benjamina L. (28 indivíduos), devido o porte da espécie não ser adequado ao passeio, que muitas vezes detém de um pequeno espaço para o plantio da árvore.

Em Aracaju-SE, Santos et al. (2015) verificaram um percentual de $33 \%$ em relação ao total de 3595 indivíduos avaliados, apresentando conflitos com característica de raízes superficiais causando problemas, sendo as espécies Ficus benjamina L. (fícus), Terminalia catappa L. (amendoeira) e a Prosopis juliflora (Sw.) DC. (algaroba) as mais conflitantes.

\section{CONCLUSÃO}

O inventário constatou que boa parte dos indivíduos que compõe a arborização urbana da região central de Imperatriz estão representados por poucas espécies, sendo as mais predominantes Licania tomentosa Benth. (Fritsch.) e Azadirachta indica A. Juss., tanto na ZC como na ZRC. Além disso, dentre as indivíduos arbóreo-arbustivos encontradas em ruas e avenidas da região central da cidade, $52 \%$ apresentaram conflitos com a fiação, construção e elementos urbanos, sendo necessária a implantação de um planejamento prévio que abranja desde a escolha correta das espécies e manutenção das mesmas em todo o seu ciclo de vida.

O estudo demonstrou ainda, que espécies como Licania tomentosa Benth. (Fritsch), Azadirachta indica A. Juss., Terminalia catappa L., Ficus benjamina L. e Mangifera indica L. não devem ser mais empregadas na arborização da cidade, visto que há um número acima do recomendado dessas espécies, além do envolvimento das mesmas na maioria dos conflitos encontrados em ruas e avenidas da Zona Central e Residencial Central de Imperatriz. 


\section{REFERÊNCIAS}

ALMEIDA, J. R.; BARBOSA, C. G. Diagnóstico da arborização urbana da cidade de CacoalRO. Revista da Sociedade Brasileira de Arborização Urbana, Piracicaba-SP, v.5, n.1, p.6181, 2010.

BARBOSA, R. P.; PORTELA, M. G. T.; MACHADO, R. R. B.; SÁ, A. S. Arborização da avenida deputado Ulisses Guimarães, bairro Promorar, zona sul de Teresina-PI. Revista da Sociedade Brasileira de Arborização Urbana, Piracicaba-SP, v. 10, n. 2, p. 78-89, 2015.

BARROS, E. F. S.; GUILHERME, F. A. G.; CARVALHO, R. S. Arborização urbana em quadras de diferentes padrões construtivos na cidade de Jatai. Revista Árvore, Viçosa, v. 34, n. 2, p. 287-295, 2010.

BOBROWSKI, R. Estrutura e dinâmica da arborização de ruas de Curitiba, Paraná, no período 1984-2010. 144 f. 2011. Dissertação (Mestrado em Engenharia Florestal)-Setor de Ciências Agrárias, Universidade Federal do Paraná, Curitiba, 2011.

BRUN, F. G. K. FUCHS, R. H.; BRUN, E. J.; ARAÚJO, L. E. B. Legislações Municipais do Rio Grande do Sul Referentes à Arborização Urbana-Estudo de Casos. Revista da Sociedade Brasileira de Arborização Urbana, Piracicaba-SP, v. 3, n. 3, p. 44-64, 2008.

CARVALHO, J. A.; NUCCI, J.C; VALASKI, S. Inventário das árvores presentes na arborização de calçadas da porção central do bairro Santa Felicidade - Curitiba/PR. Revista da Sociedade Brasileira de Arborização Urbana, Piracicaba-SP, v.5, n.1, p.126-143, 2010.

COMUNE, M. D.; SURIANI-AFFONSO, A. L. Análise de três áreas verdes urbanas em Guarapuava, Paraná. Ambiência, Guarapuava-PR, v.10, n.3, p.723 - 739, 2014.

DA FONSECA BORGES, C. A. R.; MARIM, G. C.; RODRIGUES, J. E. C. Mapeamento da cobertura vegetal do bairro da Marambaia-Belém/PA. Revista da Sociedade Brasileira de Arborização Urbana, São Paulo, n.4, p.16-26, 2012.

DE PAIVA, A. V. Aspectos da arborização urbana do Centro de Cosmópolis-SP. Revista da Sociedade Brasileira de Arborização Urbana, Piracicaba-SP, v.4, n.4, p.17-31, 2009.

DE SOUZA, P. F. I.; BOURSCHEID, C. B.; POMPÉO, P. N; STANG, M. B.; MANFROI, J. RODRIGUES, M. D. S.; DA SILVA, A.C.; HIGUCHI, P. inventário e recomendações para a arborização do centro da cidade de São Joaquim, SC. Revista da Sociedade Brasileira de Arborização Urbana, Piracicaba-SP,v. 9, n. 4, p. 99-112, 2015.

INSTITUTO BRASILEIRO DE GEOGRAFIA E ESTATÍSTICA (IBGE). Censo demográfico Imperatriz-MA. Disponível em: <http://cidades.ibge.gov.br/xtras/perfil. php?codmun=210530> Acesso em: 28 mai. 2017.

MASCARÓ, L; MASCARÓ, J. Vegetação urbana. 2.ed. Porto Alegre: Mais Quatro Editora, 2005. $204 \mathrm{p}$.

MORAES, L. A.; MACHADO, R. R. B. A arborização urbana do município de Timon/MA: inventário, diversidade e diagnóstico quali-quantitativo. Revista da Sociedade Brasileira de Arborização Urbana, Piracicaba-SP, v. 9, n. 4, p. 80-98, 2015. 
LIMA NETO, E. M.; BIONDI, D. Delineamento de unidades amostrais para o inventário da arborização de ruas em Curitiba, PR. Revista da Sociedade Brasileira de Arborização Urbana, Piracicaba -SP, v.9, n.1, p. 21 - 34, 2014.

PEREIRA, G. de A. Diagnóstico florístico da arborização urbana no Brasil e no município de Lavras - MG. 2015. 204 p. Dissertação (Mestrado em Ciências Florestais) - Universidade Federal de Lavras, 2015.

PREFEITURA DE IMPERATRIZ. A cidade. Imperatriz: Portal da Prefeitura de Imperatriz, 2016. Disponível em:<http://www.imperatriz.ma.gov.br/cidade/>. Acesso em: 14 out, 2016.

ROMANI, G. de N. Análise quali-qualitativa e por meio de sistema de informações geográficas da arborização do quadrilátero central de Ribeirão Preto. 2014. 112 f. Tese (Doutorado em Produção Vegetal) - Faculdade de Ciências Agrárias e Veterinárias - UNESP, São Paulo, 2014.

SALVI, L. T.; HARDT, L. P. A.; ROVEDDER, C. E.; FONTANA, C. S. Arborização ao longo de ruas-Túneis Verdes - em Porto Alegre, RS, Brasil: avaliação quantitativa e qualitativa. Revista Árvore, Viçosa-MG, v. 35, n. 2, p. 233-243, 2011.

SAMPAIO, A. C. F.; DE ANGELIS, B. L. D. Inventário e análise da arborização de vias públicas de Maringá-PR. Revista Sociedade Brasileira de Arborização Urbana, Piracicaba-SP, v.3, n.1, p.37-57, 2008.

SANTOS, C. Z. A.; FERREIRA, R. A.; SANTOS, L.R.; SANTOS, L. I.; GOMES, S. H.; DA GRAÇA, D. A. S. Análise qualitativa da arborização urbana de 25 vias públicas da cidade de Aracaju-SE. Ciência Florestal, Santa Maria-RS, v. 25, n. 3, p. 751-763, 2015. 